\title{
Numerical Solution of Nonlinear Systems of Algebraic Equations
}

\author{
Kamoh Nathaniel Mahwash', Gyemang Dauda Gyang ${ }^{2}$ \\ ${ }^{1}$ Department of Mathematics and Statistics, Bingham University, Karu, Nigeria \\ ${ }^{2}$ Department of Mathematics and Statistics, Plateau State Polytechnic, Barkin Ladi, Nigeria
}

Email address:

mahwash1477@gmail.com (K. N. Mahwash)daudagyemang@yahoo.com (G. D. Gyang)

\section{To cite this article:}

Kamoh Nathaniel Mahwash, Gyemang Dauda Gyang. Numerical Solution of Nonlinear Systems of Algebraic Equations. International Journal of Data Science and Analysis. Vol. 4, No. 1, 2018, pp. 20-23. doi: 10.11648/j.ijdsa.20180401.14

Received: January 29, 2018; Accepted: February 27, 2018; Published: March 23, 2018

\begin{abstract}
Considered in this paper are two basic methods of approximating the solutions of nonlinear systems of algebraic equations. The Steepest Descent method was presented as a way of obtaining good and sufficient initial guess (starting value) which is in turn used for the Broyden's method. Broyden's method on the other hand replaces the Newton's method which requires the use of the inverse of the Jacobian matrix at every new step of iteration with a matrix whose inverse is directly determined at each step by up-dating the previous inverse. The result obtained by this method revealed that the setbacks encountered in computing the inverse of the Jacobian matrix at every step number is eliminated hence saving human effort and computer time. The obtained results also showed that the number of steps that is reduced when compared to Newton's method used on the same problem.
\end{abstract}

Keywords: Convergent, Jacobian; Matrix, Approximation, Starting Value, Iteration, Nonlinear System

\section{Introduction}

Numerical solution of algebraic equations is one of the main aspects of computational mathematics. Numerical computation involves numerical answers given numerical inputs. Such problem arises whenever mathematics is used to model the real world problems (See, De Cezaro, 2008).

It is by no means uncommon for systems of nonlinear equations to occur in practice. Systems of nonlinear equations or simply nonlinear equations are equations whose variables are either of degree greater than 1 or less than 1 , but never 1.

Nonlinear equations arise in all branches of sciences and engineering. It is widely used in optimization problems and electrical circuits. There are few nonlinear equations that are readily solvable but these are definitely of the minority. Where no simple method exists for solving nonlinear equations, numerical methods are frequently employed and it is the purpose of this work to investigate some of these techniques. Gilberto (2004) presented methods for the solution of single non-linear equations as well as for systems of such equations using Newton-Raphson iteration method.
Goh and McDonald (2015) discovered that, an exact line search at a point, far from the solution, may be counterproductive.

Numerical techniques often make use of a process known as iteration. Solutions to these systems of equations $\left[\mathrm{F}_{\mathrm{i}}(\mathrm{x})=\right.$ $0 ; \mathrm{i}=1,2,3,4, \ldots, \mathrm{n}]$, are a problem that is avoided when possible, because no simple method (analytical) is found yet for solving these systems of equations. Hence this work is concerned with the numerical solutions of systems of nonlinear algebraic equations by steepest Descent and Broyden's methods. Some simple examples are solved as a perquisite to each of these methods.

The methods for solving a nonlinear system of algebraic equations of the type, $F_{i}\left(x_{j}\right)=0, i, j=1,2, \ldots, n$, dates back to the seminal work of Isaac Newton. Nowadays a Newtonlike algorithm is still the most popular; this is due to its easy numerical implementation (See Vincent and Grantham 1997, Barbashin and Krasovskii 1952 and Powell 1986). However, this type of algorithm is sensitive to the initial guess $x^{0}=$ $\left(x_{1}^{0}, x_{2}^{0}, \ldots, x_{n}^{0}\right)^{T}$ of the solution and is expensive in the computations of the Jacobian matrix $\frac{\partial F i}{\partial x j}$ and its inverse at each iterative step (See Chein-Shan \& Satya 2008 and Powell 1986). 
This work is aimed at closing the gap created by the problem of initial guess and the problem of finding the inverse of the Jacobian matrix at every iteration step encountered by Newton's methods. Though Steepest Descent methodsserve the purpose of providing sufficiently accurate initial guess, butit will always converge even for poor initial guess (See De Cezaro 2008, Huang 2011 and Powell 1970)

The idea behind Broyden's method is to compute the whole Jacobian matrix and its inverse only at the first iteration and to do a rank-one update at the other iteration steps while avoiding the inverse at each stage of the iteration (See LaSalle 1976, Gomes \& Martinez 1992 and Goh 1994).

\section{Methodology}

Consider the solution of system of nonlinear algebraic equations for which we do not know a simple analytical technique of the form

$$
f(x)=0
$$

Given the function $f$ is in the case ofn variables $x_{1}, x_{2}, x_{3}, x_{4}, \ldots, x_{n}$ which make the functions

$f_{1}\left(x_{1}, x_{2}, x_{3}, x_{4}, \ldots, x_{n}\right), f_{2}\left(x_{1}, x_{2}, x_{3}, x_{4}, \ldots, x_{n}\right), f_{3}\left(x_{1}\right.$, $\left.x_{2}, x_{3}, x_{4}, \ldots, x_{n}\right), \ldots, f_{n}\left(x_{1}, x_{2}, x_{3}, x_{4}, \ldots, x_{n}\right)$ all zero. This system of equations can be written in vector form as

$$
\mathrm{F}(X)=0
$$

Where $F=\left[f_{1}, f_{2}, . . . . ., f_{n}\right]$ and $X=$ $\left[x_{1}, x_{2}, x_{3}, x_{4}, \ldots, x_{n}\right]$. The methods of solution for one equation and one unknown will be adopted in this work.

\section{Steepest Descent Method}

This method is used to find sufficiently accurate starting approximations for the Newton-based and other techniques (See Fan and Yuan, 2005). The method determines a local minimum for a multivariable function of the form $g: R^{n} \rightarrow R$. Although the method is valuable, quite apart from the application as starting method for solving nonlinear systems, the connection between the minimization of a function from $R^{n}$ to $R$ and the solution of a system of nonlinear equation is due to the fact that the system of the form $\mathrm{F} \quad(x)=0$, has a solution at $X=$ $\left(x_{1}, x_{2}, x_{3}, x_{4}, \ldots, x_{n}\right)^{T}$ precisely when the function $g$ defined by

$$
g\left(x_{1}, x_{2}, x_{3}, x_{4}, \ldots, x_{n}\right)=\sum_{k=0}^{n}\left[f_{k}\left(x_{1}, x_{2}, x_{3}, x_{4}, \ldots, x_{n}\right)\right]^{2}
$$

has the minimal value zero.

The method of Steepest Descent for finding a local minimum for an arbitrary function $g$ from $R^{n}$ into $R$ can be intuitively be described as follows;

1. Evaluate $g$ at an initial approximation $q_{0}=$ $\left(q_{1}^{0}, q_{2}^{0}, \ldots, q_{n}^{0}\right)^{t}$

2. Determine a direction from $q_{0}$ that result in a decrease in the value of $g$.

3. Move an approximate amount in this direction and call it the new vector $q_{1}$.

4. Repeat step 1 through 3 with $q_{0}$ replaced by $q_{1}$

To extend this result to multi-variable functions, we need the following steps and definition.

$$
\text { Step 1: compute } m\left(q_{0}\right)=\sum_{k=1}^{n}\left[f_{k}\left(q_{0}\right)\right]^{2}
$$

Step 2: Compute $\nabla m\left(q_{0}\right)=2\left[J\left(q_{0}\right)\right]^{t} F\left(q_{0}\right)=A_{0}=$

$$
\left(q^{1}, q^{2}, \ldots, q^{n}\right)
$$

Step 3: Compute $H_{0}=\left\|A_{0}\right\|_{2}=\sqrt{\sum_{k=1}^{n}\left(q^{k}\right)^{2}}$

$$
\text { Step 4: Compute } H=\frac{A_{0}}{H_{0}}=\frac{1}{H_{0}}\left(A_{0}\right)
$$

Step 5: Taking $B_{1}=0, B_{2}=\frac{B_{3}}{2}=\frac{1}{2}=0.5$ and $B_{3}=1$

$$
\text { Compute } m_{2}=m\left(q_{0}-B_{2} H\right)
$$

If $m_{3} \geq m_{1}$, we reduces the seize of $B_{3}$ by $\operatorname{setting} B_{3}=\frac{B_{3}}{2}$, if $m_{3} \geq m_{1}$ still we reduce $B_{3}$ by setting $B_{3}=\frac{B_{3}}{2^{2}}$, until such a time we have $m_{3} \geq m_{1}$

$$
\begin{aligned}
& \text { Step 6: Compute } m_{3}=m\left(q_{0}-B_{3} H\right) \\
& \text { Step 7: } h_{1}=\frac{\left(m_{2}-m_{1}\right)}{B_{2}} \\
& \text { Step 8: } h_{2}=\frac{\left(m_{3}-m_{2}\right)}{B_{3}-B_{2}} \\
& \text { Step 9: } h_{3}=\frac{\left(h_{2}-h_{1}\right)}{B_{3}} \\
& \text { Step 10: } B_{0}=0.5\left(B_{2}-\frac{h_{1}}{h_{3}}\right) \\
& \text { Step 11: } m_{0}=m\left(q_{0}-B_{0} H\right) \\
& \text { If } m_{0}<m_{3}, \text { set B }=B_{0}
\end{aligned}
$$$$
\text { Step 12: Compute } q_{1}=\left(q_{0}-B H\right)
$$

\section{Brodyen's Method}

This method is one of the most effective algorithms for solving nonlinear systems of equations when the number of equations and unknowns is very large memory less implementations of this method is frequently used (See Byrd and Nocedal, 2004)

This method only requires $\mathrm{n}$ scalar functional evaluations per iteration and also reduces the number of arithmetic calculations to $\mathrm{O}\left(n^{2}\right)$. This method belongs to a class known as least change Secant updates that produces algorithms called quasi- Newton. This method replaces the Jacobian matrix in Newton's method with an approximation matrix that is updated at each iteration step (See Goh, 2010).

Suppose that an initial approximation $q_{0}$ is given to the solution of $F(x)=0$. We calculate the next approximation $q_{1}$ in the same manner as Nwetons method.

$$
\begin{aligned}
& \text { Step 1: find } A_{0}=J\left(q_{0}\right) \\
& \text { Step 2: find } A_{0}^{-1}=\left[J\left(q_{0}\right)\right]^{-1} \\
& \text { Step 3: find } \mathrm{F}\left(q_{0}\right) \\
& \text { Step 4: find } A_{0} \mathrm{~F}\left(q_{0}\right) \\
& \text { Step 5: find } q_{1}=q_{0}-A_{0} \mathrm{~F}\left(q_{0}\right)
\end{aligned}
$$

Step 6: To find $q_{2}$, we know that $q_{2}=q_{1} A_{1}^{-1} \mathrm{~F}\left(q_{1}\right)$ 
Step 7: find $A_{1}^{-1}=A_{0}^{-1}+\left[\frac{\left(S_{1}-A_{0}^{-1} y_{1}\right) S_{1}^{t} A_{0}^{-1}}{S_{1}^{t} A_{0}^{-1} y_{1}}\right]$, with $y_{1}=$ $\mathrm{F}\left(q_{1}\right)-\mathrm{F}\left(q_{0}\right)$ and $S_{1}=q_{1}-q_{0}$

Step 8: find $A_{1}^{-1} F\left(q_{1}\right)$

Step 9: find $q_{2}=q_{1} A_{1}^{-1} F\left(q_{1}\right)$

Generally, to find subsequence approximation, steps 6-9 are followed using the equation $\quad q_{k+1}=q_{k}-A_{k}^{-1} F\left(q_{k}\right) \quad$ and $\quad A_{k}^{-1}=A_{k-1}^{-1}+$ $\left[\frac{\left(S_{k}-A_{k-1}^{-1} y_{k}\right) S_{k}^{t} A_{k-1}^{-1}}{S_{k}^{t} A_{k-1}^{-1} y_{k}}\right]$ for $k \geq 1$

\section{Numerical Illustrations and Results}

In this Section, to achieve the validity, the accuracy and support the theoretical discussion of the proposed methods; the computations, associated with the examples, are performed using Scientific Workplace 5.0. Furthermore, the performance of the methods is tested on some numerical examples contained in the literature.

Examples

We consider the following nonlinear systems of equations

(a) $x_{1}^{2}-10 x_{1}+x_{2}^{2}+8=0$ and $x_{1} x_{2}^{2}+x_{1}-10 x_{2}+$ $8=0$

(b) $3 x_{1}-\cos \left(x_{2} x_{3}\right)-\frac{1}{2}=0$

$$
\begin{gathered}
x_{1}-81\left(x_{2}+0.1\right)^{2}+\sin x_{3}+1.06=0 \\
e^{-x_{1} x_{2}}+20 x_{3}+\frac{10 \pi-3}{3}=0
\end{gathered}
$$

Using Steepest Descent method with $X^{0}=(0,0,0)^{T}$ to find a reasonable starting value to the solution of the nonlinear systems of equation (a)

Let $f_{1}=x_{1}^{2}-10 x_{1}+x_{2}^{2}+8$ and $f_{2}=x_{1} x_{2}^{2}+x_{1}-$ $10 x_{2}+8$ With $x^{0}=(0,0)^{T}$

Step 1: compute $m\left(q_{0}\right)=\sum_{k=1}^{n}\left[f_{k}\left(q_{0}\right)\right]^{2}=46336$

Step 2: Compute $\operatorname{\nabla m}\left(q_{0}\right)=2\left[J\left(q_{0}\right)\right]^{t} F\left(q_{0}\right)=A_{0}=$ $\left[\begin{array}{l}-144 \\ -160\end{array}\right]$

Step 3: Compute $H_{0}=\left\|A_{0}\right\|_{2}=\sqrt{\sum_{k=1}^{n}\left(q^{k}\right)^{2}}=$ 215.2579848

Step 4:

Compute $H=\frac{A_{0}}{H_{0}}=\frac{1}{H_{0}}\left(A_{0}\right)=(-0.668964731,-0.743294146)$

Step 5: Taking $B_{1}=0, B_{2}=\frac{B_{3}}{2}=\frac{1}{2}=0.5$ and $B_{3}=1$

Compute $m_{3}=m\left(q_{0}-B_{3} H\right)=7.95256047$

If $m_{3} \geq m_{1}$, we reduces the seize of $B_{3}$ by setting $B_{3}=\frac{B_{3}}{2}$, if $m_{3} \geq m_{1}$ still we reduce $B_{3}$ by setting $B_{3}=\frac{B_{3}}{2^{2}}$, until such a time we have $m_{3} \geq m_{1}$

Step 6: Compute $m_{2}=m\left(q_{0}-B_{2} H\right)=45.81561794$
Step 7: $h_{1}=\frac{\left(m_{2}-m_{1}\right)}{B_{2}}=-92580.36876$

Step 8: $h_{2}=\frac{\left(m_{3}-m_{2}\right)}{B_{3}-B_{2}}=-75.72611494$

Step 9: $h_{3}=\frac{\left(h_{2}-h_{1}\right)}{B_{3}}=92504.64265$

Step 10: $B_{0}=0.5\left(B_{2}-\frac{h_{1}}{h_{3}}\right)=0.750409309$

Step 11: $m_{0}=m\left(q_{0}-B_{0} H\right)$

If $m_{0}<m_{3}$, set $\mathrm{B}=B_{0}$

Step 12:

Compute $q_{1}=\left(q_{0}-B H\right)=$

(0.501993201, 0.557770223). these are the starting values for $x_{1}$ and $x_{2}$ to be used in Broyden's method in

approximating the solution to the given nonlinear systems of equations (a).

Let $f_{1}=3 x_{1}-\cos \left(x_{2} x_{3}\right)-\frac{1}{2}, f_{2}=x_{1}-81\left(x_{2}+0.1\right)^{2}+$ $\sin x_{3}+1.06$ and $f_{3}=e^{-x_{1} x_{2}}+20 x_{3}+\frac{10 \pi-3}{3}$ with $X^{0}=$ $(0,0,0)^{T}$

Step 1: compute $m\left(q_{0}\right)=\sum_{k=1}^{n}\left[f_{k}\left(q_{0}\right)\right]^{2}=111.975$

Step 2: Compute $\operatorname{\nabla m}\left(q_{0}\right)=2\left[J\left(q_{0}\right)\right]^{t} F\left(q_{0}\right)=A_{0}=$ $\left[\begin{array}{c}-9 \\ -8.1 \\ 419.38\end{array}\right]$

Step 3: Compute $H_{0}=\left\|A_{0}\right\|_{2}=\sqrt{\sum_{k=1}^{n}\left(q^{k}\right)^{2}}=419.554$ Step 4:

Compute $H=\frac{A_{0}}{H_{0}}=\frac{1}{H_{0}}\left(A_{0}\right)=$ $(-0.0214514,-0.0193062,0.999583)$

Step 5: Taking $B_{1}=0, B_{2}=\frac{B_{3}}{2}=\frac{1}{2}=0.5$ and $B_{3}=1$

Compute $m_{3}=m\left(q_{0}-B_{3} H\right)=93.5649$

If $m_{3} \geq m_{1}$, we reduces the seize of $B_{3}$ by $\operatorname{setting} B_{3}=\frac{B_{3}}{2}$, if $m_{3} \geq m_{1}$ still we reduce $B_{3}$ by setting $B_{3}=\frac{B_{3}}{2^{2}}$, until such a time we have $m_{3} \geq m_{1}$

Step 6: Compute $m_{2}=m\left(q_{0}-B_{2} H\right)=2.535578495$

Step 7: $h_{1}=\frac{\left(m_{2}-m_{1}\right)}{B_{2}}=-218.8788$

Step 8: $h_{2}=\frac{\left(m_{3}-m_{2}\right)}{B_{3}-B_{2}}=-182.059$

Step 9: $h_{3}=\frac{\left(h_{2}-h_{1}\right)}{B_{3}}=400.937$

Step 10: $B_{0}=0.5\left(B_{2}-\frac{h_{1}}{h_{3}}\right)=0.522959$

Step 11: $m_{0}=m\left(q_{0}-B_{0} H\right)$

If $m_{0}<m_{3}$, set $\mathrm{B}=B_{0}$

Step

Compute $q_{1}=\left(q_{0}-B H\right)=$ $(0.011218202,0.010096351,-0.522740926)$, these are the starting values for $x_{1}, x_{2}$ and $x_{3}$ to be used byBroyden's method in approximating the solution to the given nonlinear systems of equation (b) Using Broyden's method with the obtained starting values we find the solution of the nonlinear systems of equations (a) and (b) respectively as shown in Tables 1 and 2.

Table 1. Showing result of example (a).

\begin{tabular}{lllll}
\hline $\boldsymbol{k}$ & $\boldsymbol{x}_{1}^{k}$ & $\boldsymbol{x}_{2}^{\boldsymbol{k}}$ & $\left|\boldsymbol{x}_{\mathbf{1}}^{\boldsymbol{k}}-\boldsymbol{x}_{\mathbf{1}}^{\boldsymbol{k}-\mathbf{1}}\right|$ & $\left|\boldsymbol{x}_{2}^{\boldsymbol{k}}-\boldsymbol{x}_{2}^{\boldsymbol{k}-\mathbf{1}}\right|$ \\
\hline 0 & 0.501993201 & 0.557770223 & 0.440607 & 0.3853 \\
1 & 0.9426 & 0.94307 & 0.04948 & 0.04803 \\
2 & 0.99208 & 0.9911 & 0.00774 & 0.00863 \\
3 & 0.99982 & 0.99973 & 0 & 0.00027 \\
4 & 1.00000 & 1.00000 & & 0 \\
5 & 1.00000 & 1.00000 & \\
\hline
\end{tabular}


Table 2. Showing result of example (b).

\begin{tabular}{lllllll}
\hline $\boldsymbol{k}$ & $\boldsymbol{x}_{1}^{k}$ & $\boldsymbol{x}_{2}^{k}$ & $\boldsymbol{x}_{3}^{k}$ & $\left|\boldsymbol{x}_{1}^{k}-\boldsymbol{x}_{1}^{k-1}\right|$ & $\left|\boldsymbol{x}_{2}^{k}-\boldsymbol{x}_{2}^{k-1}\right|$ & $\left|\boldsymbol{x}_{3}^{k}-\boldsymbol{x}_{3}^{k-1}\right|$ \\
\hline 0 & 0.011218202 & 0.010096351 & -0.522740926 & 0.488661798 & 0.009372 & 0.522740926 \\
1 & 0.49988 & $1.9468 \times 10^{-2}$ & -0.52152 & 0.00011 & $1.947 \times 10^{-2}$ & $1.220926 \times 0^{-3}$ \\
2 & 0.49999 & $8.738 \times 10^{-3}$ & -0.52317 & $2.0 \times 10^{-5}$ & $8.738 \times 10^{-3}$ & $1.65 \times 10^{-3}$ \\
3 & 0.50001 & $8.658 \times 10^{-4}$ & -0.52357 & $1.0 \times 10^{-5}$ & $8.74 \times 10^{-3}$ & $4.0 \times 10^{-4}$ \\
4 & 0.50000 & $3.939 \times 10^{-5}$ & -0.5236 & 0 & $8.66 \times 10^{-4}$ & $3.0 \times 10^{-5}$ \\
5 & 0.50000 & $5.81 \times 10^{-7}$ & -0.5236 & 0 & $8.7 \times 10^{-4}$ & 0 \\
6 & 0.50000 & $2.091 \times 10^{-7}$ & -0.5236 & 0 & $3.94 \times 10^{-5}$ & 0 \\
\hline
\end{tabular}

\section{Conclusion}

This work considered two basic methods of approximating the solutions of nonlinear systems of equations. The Steepest Descent method provided good and sufficient initial guess (starting value) for the Broyden's method. The starting values provided by Steepest Descent method were then used by Broyden's method to arrive at the approximate solution for each given systems of equations. This technique replaces the inverse of the Jacobian matrix [J] in Newton's method with a matrix $A_{k-1}$ whose inverse is directly determined at each step by up-dating the previous inverse thereby eliminating many computational steps if the problem were to be solved using Newton's method.

\section{References}

[1] Brazelton, J. (2010) Solving Nonlinear Equations Using Numerical Analysis. Math 451 Seminar, Tuskegee University

[2] Chein-Shan Liu \& Satya N. Atluri (2008) A Novel Time Integration Method for Solving A Large System of NonLinear Algebraic Equations. Technical Science Press CMES, 31(2):71-83

[3] De Cezaro, A (2008): On Steepest-Descent-Kaczmarz methods for regularizing systems of nonlinear ill-posed equations

[4] Gomes, F. A. M \& Martinez, J. M. (1992) Parallel implementations of Broyden's method, Springer link 47(34):361-366

[5] Huang, Tsung-Ming (2011): Numerical Solutions of Nonlinear Systems of Equations Department of Mathematics, National Taiwan Normal University, Taiwan E-mail: min@math.ntnu.edu.tw
[6] Gilberto E. Urroz (2004) Solution of non-linear equations

[7] Paul's online math notes: Algebra-Nonlinear system-Lamar University

[8] Goh, B. S. and McDonald, D. B. (2015) Newton Methods to Solve a System of Nonlinear Algebraic Equations. Journal of Optimization Theory and Application

[9] Vincent, T., Grantham, W. (1997): Nonlinear and Optimal Control Systems. Wiley, New York

[10] Barbashin, E. A. and Krasovskii, N. N. (1952): On the stability of a motion in the large. Dokl. Akad. Nauk. SSR 86, 453-456

[11] Powell, M. J. D. (1986): How Bad are the BFGS Methods when the Objective Function is Quadratic. Mathematics Programme 34, 34-47 8

[12] Goh, B. S. (1994) Global attractivity and stability of a scalar nonlinear difference equation. Computer and Mathematics Application 28, 101-107

[13] Fan, J. Y. and Yuan, Y. X. (2005): On the quadratic convergence of the Levenberg-Marquardt method without non-singularity assumption. Computing 74, 23-39

[14] Powell, M. J. D (1970) A Hybrid Method for Nonlinear Equations, in Numerical Methods for Nonlinear Algebraic Equations, pp. 87-114. Gordon and Breach, London

[15] Byrd, R. H., Marazzi, M. and Nocedal, J. (2004): On the convergence of Newton iterations to non-stationary points. Mathematics Programme 99, 127-148

[16] LaSalle, J. P. (1976): The Stability of Dynamical Systems. SIAM, Philadelphia

[17] Goh, B. S. (2010): Convergence of numerical methods in unconstrained optimization and the solution of nonlinear equations. Journal of Optimization and Theory Application $144,43-55$ 